\title{
Improving the Use of Simulation in Nursing Education: Protocol for a Realist Review
}

Torbjørg Træland Meum, PhD; Åshild Slettebø, PhD; Mariann Fossum, PhD

Department of Health and Nursing Science, University of Agder, Grimstad, Norway

Corresponding Author:

Torbjørg Træland Meum, PhD

Department of Health and Nursing Science

University of Agder

Box 509

Grimstad, 4898

Norway

Phone: 4794888747

Email: torbjorg.t.meum@uia.no

\begin{abstract}
Background: Nursing education has evolved in line with societal needs, and simulation-based learning (SBL) is increasingly being used to bridge the gap between practice and education. Previous literature reviews have demonstrated the effectiveness of using SBL in nursing education. However, there is a need to explore how and why it works to expand the theoretical foundation of SBL. Realist reviews are a theory-based approach to synthesizing existing evidence on how complex programs work in particular contexts or settings.
\end{abstract}

Objective: This review aims to understand how, why, and in what circumstances the use of simulation affects learning as part of the bachelor's program in nursing.

Methods: A realist review will be conducted in accordance with the realist template for a systematic review. In particular, we will identify and explore the underlying assumption of how SBL is supposed to work, that is, identify and explore program theories of SBL. The review will be carried out as an iterative process of searching, appraising, and synthesizing the evidence to uncover theoretical concepts that explain the causal effects of SBL. In the final section of the review, we will involve stakeholders in the Norwegian community in a web-based Delphi survey to ensure that the emerging theoretical framework derived from the published literature aligns with stakeholders' experience in practice.

Results: The Norwegian Centre for Research Data (project number 60415) has approved the study. We have performed an initial literature search, whereas quality appraisal and data extraction are ongoing processes.

Conclusions: The final outcome of the review is anticipated to extend the theoretical foundation for using simulation as an integrated component of the bachelor's program in nursing. Furthermore, the findings will be used to produce a briefing document containing guidance for national stakeholders in the community of simulation-based nursing education. Finally, the review findings will be disseminated in a peer-reviewed journal as well as national and international conferences.

International Registered Report Identifier (IRRID): DERR1-10.2196/16363

(JMIR Res Protoc 2020;9(4):e16363) doi: 10.2196/16363

\section{KEYWORDS}

education; nursing; learning; realist review; simulation training

\section{Introduction}

The health care sector has undergone radical changes over the last few decades, a fact that has also affected nursing education. Demographic changes, technological development, and innovation in health science have led to increasing requirements being placed on the education of health professionals. A study carried out by the Carnegie Foundation for the Advancement of Teaching [1] has explored the strengths and weaknesses of nursing education, and the authors call for a radical transformation of nursing education. In particular, they describe a gap between education and practice, that is, "the ability of a practice setting to adopt and reflect what was taught in academic institutions" [1]. The authors also highlight the 
practice-education gap "as it is becoming more and more difficult for nursing education to keep pace with the rapid changes in clinical practice driven by research and new technologies" [1]. Patient safety is the cornerstone of high-quality health care [2]. The Institute of Medicine has highlighted evidence-based practice as one of the key competences all clinicians should possess to meet the needs of 21 st-century health care services [3]. These competences have been adapted to the nursing community, which has in turn proposed statements on the knowledge, skills, and attitudes that should be developed during prelicensure nursing education [4]. The ability to integrate scientific knowledge into practice requires good clinical judgment and reasoning, which is considered an essential part of nursing. Clinical judgment in nursing has been defined as "interpretation or conclusion about a patient's needs, concerns, or health problems, and/or the judgement to take action (or not), use or modify standard approaches, or improvise new ones as deemed appropriate by the patient's response" [5]. From this point of view, clinical reasoning refers to the processes by which nurses and other clinicians arrive at their judgment, including analytical processes, intuition, and narrative thinking [5]. Similar research has also pointed to the situated, practice-based aspect of clinical reasoning and emphasized the ability to discern the relevance of the evidence behind general scientific knowledge and how it applies to a particular clinical situation [6]. The rapid expansion of clinical knowledge over the past decades has led to increasing demands on health professionals to employ evidence-based practice [3]. Clinical judgment is a capability that evolves in line with clinical experience, and the challenge is to integrate clinical knowledge into classroom teaching [7].

Simulation-based learning (SBL) has in recent years been increasingly utilized to bridge the gap between practice and education. The International Nursing Association for Clinical Simulation and Learning has defined simulation as "an educational strategy in which a particular set of conditions are created or replicated to resemble authentic situations that are possible in real life" [8]. The use of simulation in the education of health professionals is not a new innovation; however, the evolving use of advanced technology in recent decades has provided new capabilities for the educational use of simulation. Some of the early versions of full-body patient simulators were designed for subject-specific areas. For example, a Norwegian company developed Resuci Anne in 1960 for cardiopulmonary resuscitation training $[9,10]$. Since then, full-scale patient simulators that facilitate the formation of dynamic patient situations that fully mirror actual clinical settings have become available [11]. Accordingly, a number of simulation modalities, such as full- and part-body models with low- and high-tech features, computer-based programs, and standardized patients, have been used as an educational resource in health care [11]. Since then, simulation has gradually been integrated into the education of health professionals as an educational intervention that includes digital technologies, human resources, and educational strategies. Experiential learning [12] and situated learning theory [13] have inspired the design and development of simulated learning activities in nursing [14]. These theories emphasize active engagement in the learning process, in which knowledge is created through the transformation of experience.
All in all, advancement in educational principles and digital technology has provided the opportunity to create realistic learning activities in a safe environment and, thus, bridge the gap between the classroom and clinical practice.

A growing body of evidence in the simulation literature has demonstrated the effectiveness of simulation in the teaching of clinical knowledge, procedural skills, and teamwork [11,15]. A systematic literature review by Lapkin et al [16] showed that the use of patient simulation manikins in teaching improved knowledge acquisition, critical thinking, and the ability to identify deteriorating patients. Similarly, a recent literature review conducted by Cant and Copper [17] revealed positive outcomes of simulation education for knowledge acquisition, psychomotor skills, self-efficiency, satisfaction, confidence, and critical thinking. Virtual patient simulation is also increasingly used in nursing education. A randomized controlled trial by Liaw et al [18] compared virtual simulation with manikin-based simulation and demonstrated that both the simulation modalities were effective learning strategies for improving nursing students' clinical performance. Although previous research has shown promising effects for using simulation as an educational intervention, studies have also called for a deeper theoretical understanding of how and why simulation contributes to learning [19-22].

The realist review is a method that is increasingly used in health care to explore "What works for whom, in what circumstances and why?" [23-25]. The method is based on a realist philosophy of science, which positions itself between positivism and constructivism [23]. Basically, a realist approach recognizes the existence of an external social reality. However, there is a social reality that cannot be measured directly because our knowledge of it is processed through our brains, human senses, language, and culture. The realist review was developed as a theory-based approach to synthesizing existing evidence on how complex programs work in particular contexts or settings. The unit of analyses is to identify, test, and refine the program theory or theories, that is, the underlying assumptions about how an intervention is supposed to work [24]. The analytical process in realist review emphasizes the causal relationship between contexts, mechanisms, and outcomes (CMO). This process is described as the CMO configuration where the concept of mechanism is considered as the causal force that operates in a particular context to generate the desired outcome. All in all, the analytical process involves iterative testing of the $\mathrm{CMO}$ configurations and refinement of the theoretical concepts derived from relevant evidence (qualitative, quantitative, and mixed method studies) [24].

Realist reviews have been used in health care as an approach to the synthesis of evidence in various disciplines such as implementation science [26] and internet-based medical education [27]. Similarly, McGaghie et al [28] used a combined critical and realist review methodology to evaluate research on simulation-based education and highlighted 12 features and best practices to promote the educational impact of using simulation. Research on SBL in nursing education is extensive; however, realist reviews have rarely been used in this research community. Accordingly, we consider a realist review to be a promising method to uncover the causal effects of SBL and address the 
call for more theory-driven research to promote the educational use of simulation in nursing education.

\section{Methods}

\section{The Purpose of the Review}

The overall purpose of this review is to gain a deeper theoretical insight into how the use of simulation affects learning to promote the educational use of simulation as an integral part of the bachelor's program in nursing. It is part of a larger research project focusing on professional development at the department of nursing of a large regional university in Norway. Moreover, the project is affiliated with a research group at the department of nursing science (health care services, ethics, and quality) that highlights innovative and practice-based research to educate future-oriented nurses. The cocreation of knowledge is one of this university's overarching visions as it strives to develop and implement future-oriented, varied, student-centered, and practice-based teaching and learning methods at all levels of instruction. In accordance with this vision, SBL has for many years been an established method of learning as a supplement to traditional teaching. On the basis of challenges, such as an increasing intake of students, it is anticipated that SBL will be used to an even greater extent as an integrated part of the curriculum.

\section{Research Aim}

This review aims to identify how the use of simulation affects learning in the bachelor's program in nursing.

\section{Research Question}

The research conducted in this study will explore what sort of SBL "works" for whom and in what circumstances. The study

Figure 1. Review design. will supplement and expand upon previous research in this field, exploring the following questions:

1. How does the use of SBL affect the development of clinical reasoning, clinical judgment, and skills in nursing education?

2. How are the principles of learning translated into learning activities in the simulation setting?

3. What characterizes conditions in the simulation environment that facilitate learning in the bachelor's program in nursing?

\section{Objectives}

The research objectives of the study are as follows:

1. To conduct a realist review to understand how, why, and in what circumstances the use of simulation affects learning as part of the bachelor's program in nursing.

2. To identify and refine program theories for SBL.

3. To make recommendations with regard to how the potential for using simulation may be utilized in the bachelor's program in nursing.

\section{Study Design}

A systematic literature review inspired by the realist approach to evaluation will be carried out [29]. The use of a realist review is a relatively new strategy for synthesizing research and methodological guidance, and training materials have been developed by an interdisciplinary research team [24]. The review process mainly follows the same steps as traditional systematic reviews. However, the unit of analysis in a realist approach is the refinement of program theory, which is characterized as an iterative process that includes both qualitative and quantitative studies [25]. Accordingly, we will utilize the realist template for systematic review [25] and follow the steps illustrated in Figure 1.
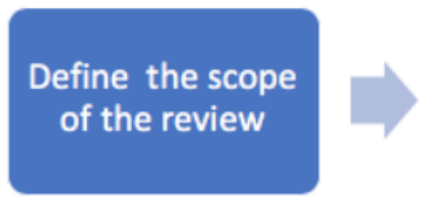

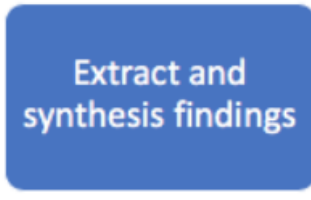

Draw conclusions

and make

recommendation

\section{Stage 1: Defining the Scope of the Review}

The first step is to refine the purpose of the review and identify candidate theories, that is, the theoretical basis for why SBL works $[24,27]$. Two researchers will take part in every step of the process, and one of the team members has extensive knowledge of SBL. On the basis of our knowledge of previous research on the use of SBL, we have acknowledged the need to explore how, why, and for whom it works. Our initial research questions will be followed with a special emphasis on what sorts of SBL work for whom and in what circumstances. The objective is to elucidate what and how SBL promotes clinical judgment and clinical skills in the bachelor's program in nursing and then explain this elucidation through the use of middle-range theory. A key point at this stage is to identify existing theories regarding the use of SBL in the bachelor's program in nursing.

Previous research has identified Jeffries Framework [30], Kolb's theory of experiential learning [12], and Bandura's social cognitive theory [31] as the most frequently used frameworks and theories in SBL [22]. These theories are characterized as middle-range theories (theories that can usefully be applied to a family of interventions) [24]. These theories, therefore, provide a starting point in the process of identifying program theories that strive to explain the chain of events underlying SBL. As this is an iterative process, during the search and screening stages, we will attempt to expand the list of relevant middle-range theories.

\section{Stage 2: Searching For and Appraising the Evidence}

The search strategy will follow the guidelines provided by Booth et al [32] and include six relevant web-based databases: Cochrane Library, Cumulative Index of Nursing and Allied 
Health Literature, Medical Literature Analysis and Retrieval System Online, EMBASE, Education Resources Information Center, and Web of Science. The search for evidence will be carried out in collaboration with a health science librarian. Furthermore, it will mainly include the following search terms: "Nursing students," Nursing education," Baccalaureate," "Simulation," "Simulated environment," "Simulation training," "Manikin," and "Anatomic model." Each search will use the relevant search terms or MESH/thesaurus/keyword heading for each database. As indicated by our research questions, we will focus on outcomes related to clinical judgment, clinical reasoning, and clinical skills. However, we have not included these terms in our database search because of their ambiguity, which may lead to the exclusion of relevant studies. To ensure sensitivity, we will do a manual screening with these terms as part of the inclusion criteria. SBL is a comprehensive field of study, and we need to make some pragmatic decisions for which studies we want to include and exclude. Simulation in health care is usually classified in terms of fidelity, that is, the level of realism associated with a particular simulation activity [33]. However, the concept of "fidelity" is not clearly stated in the literature [34], and we will use the definitions drawn up by the Society for Simulation in Healthcare to distinguish between the physical, psychological, and environmental aspects of fidelity [33]. Recent literature reviews have mainly focused on high-fidelity simulation in laboratory settings using computerized full-body manikins [35]. Although high-fidelity simulators are considered a useful learning resource, McGaghie et al [28] have emphasized the link between educational goals and simulation tools as a key principle for the effective use of simulation. Thus, we will include medium- and low-fidelity simulators in the initial screening as we consider these to be the most appropriate in teaching basic nursing. In addition, we want to identify learning outcomes using Kirkpatrick's 4 levels of evaluation: (1) reaction, (2) learning, (3) behavior, and (4) results [36]. Accordingly, our initial inclusion and exclusion criteria are shown in Textboxes 1 and 2 .

Textbox 1. Inclusion criteria for this study.

- Bachelor's degree in nursing

- $\quad$ Focus on the student's perspective

- $\quad$ Medium- and low-fidelity simulators

- $\quad$ Every phase of the simulation process included

- Clinical judgment, clinical reasoning, and clinical skills

- Learning outcome related to at least level 2 of the training evaluation described by Kirkpatrick

- $\quad$ Peer-reviewed research paper (qualitative, quantitative, and mixed method studies) written in English

- $\quad$ Published between 2014 and 2019

Textbox 2. Exclusion criteria for this study.

- $\quad$ Continuing education (nurse practitioner, advanced nursing, and midwife)

- High-fidelity simulators, serious games, electronic learning, and virtual reality

- Comparison of different simulation methods

- Interdisciplinary simulation

- Disaster management

- $\quad$ Review articles and doctoral thesis

The preliminary criteria have been developed for searching and identifying middle-range theories that explain how and why SBL works (or does not work). In accordance with the recommendations for realist reviews, we will include qualitative, quantitative, and mixed method studies. However, we will not conduct snowball sampling or include "grey" literature such as reports, theses, or conference papers.

Search results will be saved as text files and downloaded into a web-based software platform (Covidence) for screening and quality appraisal. Two members of the review team will screen the title and abstract with respect to the inclusion and exclusion criteria. A full-text screening and quality assessment will then be performed based on quality appraisal criteria for realist reviews, that is, relevance (whether it can contribute to theory building and/or testing) and rigor (whether the methods used to generate the relevant data are credible and trustworthy) [24]. In addition, we will use the criteria described by Dixon-Woods et al [37] as well as the Mixed Methods Appraisal Tool [38] to aid our decision making on methodological credibility. When agreement is reached, data will be converted to a flowchart to illustrate the search and screening process as well as the final selection of included papers. References and data derived from the quality assessment will first be stored in Covidence and then transferred to a Microsoft Excel spreadsheet.

\section{Stage 3: Data Extraction and Synthesizing the Results}

At this stage, we will transfer both papers and bibliographical data to NVivo software (QSR International) for further data extraction and syntheses. First and foremost, the characteristics 
of the included studies will be listed in a table. One of the advantages of NVivo is its ability to transfer bibliographical data to a classification sheet when a library is imported from a reference management software program, for example, Mendeley. Descriptive information on the included studies (titles, authors, sources, and publication year) will, thus, be transferred to the classification sheet in NVivo, which will form the starting point for further data extraction. On the basis of previous realist reviews [39], we will add data domains to the classification sheet related to research design, educational setting, educational consequences, and outcome measures. Furthermore, we will use NVivo to code section of texts that may prove useful for constructing theory and refining theoretical concepts. Generative mechanisms (mechanism of action) are considered to be the key unit of analysis, and data coding will involve identifying the interrelations between CMO in the included studies. Data extraction and synthesis are considered to be an interwoven process where "raw data" captured in the included studies will be used to make sense of the causal relationship between CMO identified in the included studies.

Data synthesis will follow the realist review guidelines through using an interpretive approach to data synthesis [24]. To make sense of our "raw" data, we will incorporate the concepts identified in the primary studies into a higher-order theoretical structure [40]. First of all, we will uncover any semirecurring patterns of behavior (demi-regularities) that may be present in the included studies. We will then explore if our initial middle-range theories are able to explain why these demi-regularities emerge under the contexts reported in our included papers [39]. The middle-range theories will be treated as theoretical data and included in a constant comparison of key demi-regularities derived from the included studies with emerging theoretical conceptualization [24]. The main objective of the review process is to refine the program theory, which may involve several iterations that include testing and refining to progress toward a refined theoretical framework of SBL in nursing education.

\section{Stage 4: Drawing Conclusions and Making Recommendations}

The findings will provide theoretical and practical implications for SBL in nursing education. The inductive analysis and synthesis of data extracted from the included studies have the potential to generate theoretical concepts that explain the causal effect of SBL. Uncovering the generative mechanisms provides us with the ability to form a preliminary theoretical framework in our efforts to refine the program theory of SBL [29]. In line with similar reviews, the findings will be transformed into recommendations that can be used to inform policy makers and practitioners. The intended outcome is that practitioners will take note of the findings and implement them. Thus, we expect the review's findings to influence the design of new programs, at this stage involving practitioners. Stakeholders' practical knowledge will be used in the refinement of the program theory to ensure that the emerging theoretical framework derived from the published literature aligns with stakeholders' experiences in practice.
Stakeholders will mainly be involved through using a web-based Delphi method. This is a well-known method for giving structure to group processes used to identify problems, setting goals and priorities, and identifying problems as well as solutions [41]. The method has evolved into a group-facilitating technique in health and social care. It contains an iterative multistage process designed to transform opinion into group consensus [42]. The method's democratic, structured approach that utilizes participants' collective knowledge is considered to be a key advantage [43], having the potential to broaden the knowledge within the nursing profession [42]. The Delphi method has been used in various settings for different purposes; in this particular case, we will apply the research-based guidelines outlined by Hasson et al [42] and Okoli and Pawlowski [44] to maintain validity and credibility in the research process. Selecting qualified experts (a panel of informed individuals) plays a key role in this process. Therefore, our preparations will include using an Excel spreadsheet as a tool to categorize and identify relevant experts, that is, knowledgeable and experienced professionals in SBL. This study will include the Norwegian community, and we will attempt to get input from a wide range of simulation experts comprising associate professors, researchers, and members of professional networks. Furthermore, we will identify relevant panelists from various organizational units such as universities, the Norwegian Nurses Organisation, and "MedSimNorge" (the national network for simulation in the health care sector). Potential participants will be identified by gatekeepers in relevant organizations, and we aim to recruit about 35 participants to the panel. Furthermore, the literature synthesis' key findings will be transformed into statements intended as briefing material for the panelists. In accordance with the guidelines for the Delphi technique, we will carry out several rounds of online questionnaires (brainstorming, narrowing, and ranking) until consensus is reached [44]. The briefing document, as well as three or four additional questions, will be sent to the participants in the process' first round. Subsequent rounds will involve validating, refining, and ranking the statements from previous rounds. The goal of the final phase is to reach consensus about ranking relevant statements, and the panelist will be asked to rank each potential item on a 7-point Likert scale ( $1=$ strongly disagree to $7=$ strongly agree). The ranking results will then be collated and inserted into an Excel spreadsheet and analyzed using nonparametric statistics (Kendall W). In addition, any free-text comments from the panelist will be analyzed thematically. The findings from the Delphi survey will be summarized, a process that involves incorporating the stakeholders' points of view into the final part of the analytical process.

The steps in the review process are overlapping and iterative, which means that the review steps can be revised throughout the process as new ideas and evidence emerge. We will, thus, follow an iterative process until theoretical saturation is achieved, that is, when new data do not provide any new theoretical insight into the emerging theory. Finally, the study will be reported in accordance with publication standards for realist review [45]. A diagram will be used to present the final program theory. 


\section{Ethics and Dissemination}

This study has been approved by the Norwegian Centre for Research Data (project number 60415). The review findings will be presented at national and international conferences, including the annual conference organized by the Society for Simulation in Europe. Finally, the findings will be disseminated in a peer-reviewed journal.

\section{Results}

The initial search generated 4830 unique references, and after initial screening, we have included 113 studies. Data extraction and synthesis are ongoing processes, and we plan to complete a first draft of the literature review in June 2020.

\section{Discussion}

Simulation is still a relatively new field of research, and we expect that the findings from this realist review will lead to theoretical and practical implications for the use of simulation in nursing education. Several studies have emphasized the need for conceptual and theoretical frameworks for the use of simulation [14,19], and the final outcome of this review is expected to generate theoretical concepts that may explain the causal effects of simulation as an integrated part of nursing education. The findings will then be used to produce a briefing document to guide practitioners in designing educational programs for the bachelor's degree in nursing. Although the intended audience for this review is nursing educators, we expect the findings to be relevant to other health care professionals.

In this study, we have outlined the different steps involved in a realist review, and we consider it as a promising method to unpack the complexity of SBL. The realist review method has increasingly been used across different research fields; however, it has rarely been used in simulation-based nursing education. A major advantage of the method is to move beyond the effect of using simulation toward exploration of the causal mechanisms involved in the intervention. However, there are some key issues to consider when applying the method. As previously mentioned, the realist review method is based on a realist philosophy of science [29]. Thus, it is necessary to consider the underlying ontological and epistemological assumptions of the realist philosophy to understand the methodological implications. The realist approach is regarded as a middle way between positivism and interpretivism and embraces a variety of qualitative and quantitative methods [46]. This makes it particularly appropriate for the study of SBL that incorporates aspects of both natural science and social science. A central theme of a realist approach is the power of generative mechanisms; however, it can be challenging to know how to identify and define them. To gain a broader understanding of these issues, we will incorporate recent research from other research disciplines that deal with methodological implications of critical realism [46]. We, thus, expect the study to increase the insight into methodological challenges that have been pointed out in similar research [39].

\section{Acknowledgments}

This publication is supported by the Department of Health and Nursing Science, University of Agder.

\section{Authors' Contributions}

TM designed and drafted the protocol with input from $\AA$ S and MF. All authors have read and approved the final manuscript.

\section{Conflicts of Interest}

None declared.

\section{References}

1. Benner P, Sutphen M, Leonard V, Day L. Educating Nurses: A Call For Radical Transformation. San Francisco: Jossey-bass; 2010.

2. Hughes RG. Patient Safety and Quality: An Evidence-based Handbook for Nurses. Rockville: Agency for Healthcare Research and Quality; 2008.

3. Greiner A, Knebel E. Health Professions Education: A Bridge to Quality. Washington, DC: National Academies Press; 2003.

4. Cronenwett L, Sherwood G, Barnsteiner J, Disch J, Johnson J, Mitchell P, et al. Quality and safety education for nurses. Nurs Outlook 2007;55(3):122-131. [doi: 10.1016/j.outlook.2007.02.006] [Medline: 17524799]

5. Tanner CA. Thinking like a nurse: a research-based model of clinical judgment in nursing. J Nurs Educ 2006 Jun;45(6):204-211. [doi: 10.3928/01484834-20060601-04] [Medline: 16780008]

6. Benner P, Hughes RG, Sutphen M. Clinical reasoning, decisionmaking, and action: thinking critically and clinically. In: Hughes RG, editor. Patient Safety and Quality: An Evidence-Based Handbook for Nurses. Rockville, MD: Agency for Healthcare Research and Quality (US); 2008:87-110.

7. Benner P. Curricular and pedagogical implications for the Carnegie Study, educating nurses: a call for radical transformation. Asian Nurs Res (Korean Soc Nurs Sci) 2015 Mar;9(1):1-6 [FREE Full text] [doi: 10.1016/j.anr.2015.02.001] [Medline: 25829203]

8. INACL Standards Committee. INACSL standards of best practice: SimulationSM Simulation glossary. Clin Simul Nurs 2016;12:S39-S47. [doi: 10.1016/j.ecns.2016.09.012]

9. Buck GH. Development of simulators in medical education. Gesnerus 1991;48 Pt 1:7-28. [Medline: 1855669$]$ 
10. Hayden JK, Smiley RA, Alexander M, Kardong-Edgren S, Jeffries PR. The NCSBN National Simulation Study: a longitudinal, randomized, controlled study replacing clinical hours with simulation in prelicensure nursing education. J Nurs Regul 2014;5(2):S3-40. [doi: 10.1016/s2155-8256(15)30062-4]

11. Nagle BM, McHale JM, Alexander GA, French BM. Incorporating scenario-based simulation into a hospital nursing education program. J Contin Educ Nurs 2009 Jan;40(1):18-25; quiz 26. [doi: 10.3928/00220124-20090101-02] [Medline: 19226995]

12. Kolb AY, Kolb DA. Learning styles and learning spaces: enhancing experiential learning in higher education. Acad Manag Learn Educ 2005;4(2):193-212. [doi: 10.5465/amle.2005.17268566]

13. Lave J, Wenger E. Situated Learning: Legitimate Peripheral Participation. Cambridge: Cambridge University Press; 1991.

14. Bland AJ, Topping A, Wood B. A concept analysis of simulation as a learning strategy in the education of undergraduate nursing students. Nurse Educ Today 2011 Oct;31(7):664-670. [doi: 10.1016/j.nedt.2010.10.013] [Medline: 21056920]

15. Okuda Y, Bryson EO, DeMaria S, Jacobson L, Quinones J, Shen B, et al. The utility of simulation in medical education: what is the evidence? Mt Sinai J Med 2009 Aug;76(4):330-343. [doi: 10.1002/msj.20127] [Medline: 19642147]

16. Lapkin S, Levett-Jones T, Bellchambers H, Fernandez R. Effectiveness of patient simulation manikins in teaching clinical reasoning skills to undergraduate nursing students: a systematic review. Clinical Simulation in Nursing 2010 Nov;6(6):e207-e222. [doi: 10.1016/j.ecns.2010.05.005]

17. Cant RP, Cooper SJ. Use of simulation-based learning in undergraduate nurse education: an umbrella systematic review. Nurse Educ Today 2017 Feb;49:63-71. [doi: 10.1016/j.nedt.2016.11.015] [Medline: 27902949]

18. Liaw SY, Chan SW, Chen FG, Hooi SC, Siau C. Comparison of virtual patient simulation with mannequin-based simulation for improving clinical performances in assessing and managing clinical deterioration: randomized controlled trial. J Med Internet Res 2014 Sep 17;16(9):e214 [FREE Full text] [doi: 10.2196/jmir.3322] [Medline: 25230684]

19. Issenberg SB, Ringsted C, Ostergaard D, Dieckmann P. Setting a research agenda for simulation-based healthcare education: a synthesis of the outcome from an Utstein style meeting. Simul Healthc 2011 Jun;6(3):155-167. [doi: 10.1097/SIH.0b013e3182207c24] [Medline: 21642804]

20. Graham AC, McAleer S. An overview of realist evaluation for simulation-based education. Adv Simul (Lond) 2018;3:13 [FREE Full text] [doi: 10.1186/s41077-018-0073-6] [Medline: $\underline{\text { 30026966] }}$

21. Bajpai S, Semwal M, Bajpai R, Car J, Ho AH. Health professions' digital education: review of learning theories in randomized controlled trials by the digital health education collaboration. J Med Internet Res 2019 Mar 12;21(3):e12912 [FREE Full text] [doi: 10.2196/12912] [Medline: $\underline{30860483}$ ]

22. Lavoie P, Michaud C, Bélisle M, Boyer L, Gosselin É, Grondin M, et al. Learning theories and tools for the assessment of core nursing competencies in simulation: a theoretical review. J Adv Nurs 2018 Feb;74(2):239-250. [doi: 10.1111/jan.13416] [Medline: 28815750]

23. Wong G, Greenhalgh T, Westhorp G, Pawson R. Realist methods in medical education research: what are they and what can they contribute? Med Educ 2012 Jan;46(1):89-96. [doi: 10.1111/j.1365-2923.2011.04045.x] [Medline: 22150200]

24. Wong G, Greenhalgh T, Westhorp G, Pawson R. NCBI - NIH. 2014. Development of Methodological Guidance, Publication Standards and Training Materials for Realist and Meta-narrative Reviews: The Rameses (Realist and Meta-Narrative Evidence Syntheses - Evolving Standards) Project URL: https://www.ncbi.nlm.nih.gov/pubmed/25642521 [accessed 2020-02-28]

25. Pawson R, Greenhalgh T, Harvey G, Walshe K. Semantic Scholar. 2004. Realist Synthesis: An Introduction URL: https:/ /pdfs.semanticscholar.org/4351/46e6e6617491ff1c4b32b76e0a534c86d6c7.pdf?_ga=2.132281806.1395435436. 1582877966-1679671381.1567599385 [accessed 2020-02-28]

26. Varsi C, Nes LS, Kristjansdottir OB, Kelders SM, Stenberg U, Zangi HA, et al. Implementation strategies to enhance the implementation of eHealth programs for patients with chronic illnesses: realist systematic review. J Med Internet Res 2019 Sep 27;21(9):e14255 [FREE Full text] [doi: 10.2196/14255] [Medline: 31573934]

27. Wong G, Greenhalgh T, Pawson R. Internet-based medical education: a realist review of what works, for whom and in what circumstances. BMC Med Educ 2010 Feb 2;10:12 [FREE Full text] [doi: 10.1186/1472-6920-10-12] [Medline: 20122253]

28. McGaghie WC, Issenberg SB, Petrusa ER, Scalese RJ. A critical review of simulation-based medical education research: 2003-2009. Med Educ 2010 Jan;44(1):50-63. [doi: 10.1111/j.1365-2923.2009.03547.x] [Medline: 20078756]

29. Pawson R, Greenhalgh T, Harvey G, Walshe K. Realist review--a new method of systematic review designed for complex policy interventions. J Health Serv Res Policy 2005 Jul;10(Suppl 1):21-34. [doi: 10.1258/1355819054308530] [Medline: 16053581]

30. Jeffries PR. A framework for designing, implementing, and evaluating simulations used as teaching strategies in nursing. Nurs Educ Perspect 2005;26(2):96-103. [Medline: 15921126]

31. Bandura A. Self-efficacy: toward a unifying theory of behavioral change. Psychol Rev 1977 Mar;84(2):191-215. [doi: 10.1037//0033-295x.84.2.191] [Medline: $\underline{847061]}$

32. Booth A, Sutton A, Papaioannou D. Systematic Approaches To A Successful Literature Review. Los Angeles, CA: Sage Publications Ltd; 2012. 
33. Lopreiato JO, Society for Simulation in Healthcare. Agency for Healthcare Research and Quality. Healthcare Simulation Dictionary URL: https://www.ahrq.gov/sites/default/files/publications/files/sim-dictionary.pdf [accessed 2020-02-28]

34. Schoenherr JR, Hamstra SJ. Beyond fidelity: deconstructing the seductive simplicity of fidelity in simulator-based education in the health care professions. Simul Healthc 2017 Apr;12(2):117-123. [doi: 10.1097/SIH.0000000000000226] [Medline: 28704289]

35. Cant RP, Cooper SJ. The value of simulation-based learning in pre-licensure nurse education: a state-of-the-art review and meta-analysis. Nurse Educ Pract 2017 Nov;27:45-62. [doi: 10.1016/j.nepr.2017.08.012] [Medline: 28843948]

36. Kirkpatrick DL, Kirkpatrick JD. Evaluating Training Programs: The Four Levels. Third Edition. San Francisco: Barrett Koehler Publishers Inc; 2006.

37. Dixon-Woods M, Cavers D, Agarwal S, Annandale E, Arthur A, Harvey J, et al. Conducting a critical interpretive synthesis of the literature on access to healthcare by vulnerable groups. BMC Med Res Methodol 2006 Jul 26;6:35 [FREE Full text] [doi: 10.1186/1471-2288-6-35] [Medline: 16872487]

38. Hong Q, Pluye P, Fàbregues S, Bartlett G, Boardman F, Cargo M, et al. mixedmethodsappraisaltoolpublic. Mixed Methods Appraisal Tool URL: http://mixedmethodsappraisaltoolpublic.pbworks.com/ [accessed 2019-11-30]

39. Wong G. The internet in medical education: a worked example of a realist review. In: Hannes K, Lockwood C, editors. Synthesizing Qualitative Research: Choosing the Right Approach. Hoboken, New Jersey: John Wiley \& Sons Ltd; 2012:83-112.

40. Dixon-Woods M, Agarwal S, Jones D, Young B, Sutton A. Synthesising qualitative and quantitative evidence: a review of possible methods. J Health Serv Res Policy 2005 Jan;10(1):45-53. [doi: 10.1177/135581960501000110] [Medline: $\underline{15667704]}$

41. Delbecq AL, van de Ven A, Gustafson DH. Group Techniques for Program Planning: A Guide to Nominal Groups and Delphi Process. Glenview, Illinois: Scott, Foresman and Company; 1975.

42. Hasson F, Keeney S, McKenna H. Research guidelines for the Delphi survey technique. J Adv Nurs 2000 Oct;32(4):1008-1015. [Medline: 11095242 ]

43. Powell C. The Delphi technique: myths and realities. J Adv Nurs 2003 Feb;41(4):376-382. [doi: 10.1046/j.1365-2648.2003.02537.x] [Medline: 12581103 ]

44. Okoli C, Pawlowski SD. The Delphi method as a research tool: an example, design considerations and applications. Inf Manag 2004;42(1):15-29. [doi: 10.1016/j.im.2003.11.002]

45. Wong G, Greenhalgh T, Westhorp G, Buckingham J, Pawson R. RAMESES publication standards: realist syntheses. J Adv Nurs 2013 May;69(5):1005-1022. [doi: 10.1111/jan.12095] [Medline: 23356726]

46. Zachariadis M, Scott S, Barrett M. Methodological implications of critical realism for mixed-methods research. Manag Inf Syst Q 2013;37(3):855-879. [doi: 10.25300/misq/2013/37.3.09]

\section{Abbreviations \\ CMO: contexts, mechanisms, and outcomes \\ SBL: simulation-based learning}

Edited by G Eysenbach; submitted 23.09.19; peer-reviewed by S Khan, S Sullivan, T Risling; comments to author 19.11.19; revised version received 14.01.20; accepted 08.02.20; published 29.04.20

Please cite as:

Meum TT, Sletteb $\varnothing$ A, Fossum $M$

Improving the Use of Simulation in Nursing Education: Protocol for a Realist Review

JMIR Res Protoc 2020;9(4):e16363

URL: http://www.researchprotocols.org/2020/4/e16363/

doi: $\underline{10.2196 / 16363}$

PMID: 32347808

(CTorbjørg Træland Meum, Åshild Slettebø, Mariann Fossum. Originally published in JMIR Research Protocols (http://www.researchprotocols.org), 29.04.2020. This is an open-access article distributed under the terms of the Creative Commons Attribution License (https://creativecommons.org/licenses/by/4.0/), which permits unrestricted use, distribution, and reproduction in any medium, provided the original work, first published in JMIR Research Protocols, is properly cited. The complete bibliographic information, a link to the original publication on http://www.researchprotocols.org, as well as this copyright and license information must be included. 